\title{
Data Driven Cone Beam CT Motion Management for Radiotherapy Application
}

\author{
Adeyemi Akintonde, Student Member IEEE, Jamie McClelland, Helen Grimes, Syed Moinuddin, Ricky A. Sharma, \\ Simon. Rit, Kris. Thielemans, Senior Member, IEEE
}

\begin{abstract}
The ability to identify respiratory motion is crucial during radiation therapy treatment. In our study we introduced a novel data driven method based on principal component analysis (PCA) to extract a signal related to respiratory motion from cone beam CT projection data. Projection data acquired on cone beam CT devices normally has two motion component information within it, (1) respiratory induced motion and (2) detector rotational induced motion. Our novel approach for extracting a respiratory induced motion signal from projection data was based on computing PCA for different sections of the data set independently, and introducing a technique of combining the extracted signal from each section in a manner to represent the respiratory signal from the entire data set. We tested our method using simulation data set from XCAT software and a real patient data set. The respiratory signal extracted with the XCAT simulation yielded comparable result when compared to the ground truth respiratory signal. Initial results for the real patient data set are encouraging but show need for further refinements.
\end{abstract}

\section{INTRODUCTION}

Cone Beam Computed Tomography (CBCT) imaging is $C_{\text {commonly used for patient's position verification during }}$ radiation therapy treatment, and to observe changes to anatomy and motion. CBCT acquisition takes approximately 1 minutes, and such a relatively long acquisition time causes respiratory induced motion artefact in the reconstructed

Manuscript received November 10, 2017. This work was supported by the EPSRC-funded UCL Centre for Doctoral Training in Medical Imaging (EP/L016478/1) and the Department of Health's NIHR-funded Biomedical Research Centre at University College London Hospitals.

Ricky A. Sharma is supported by the NIHR University College London Hospitals Biomedical Research Centre

Adeyemi Akintonde is with the Institute of Nuclear Medicine, University College London. He is also with the Department of Medical Physics \& Biomedical Engineering, London NW1 2BU, UK (e-mail: adeyemi.akintonde.15@ucl.ac.uk ).

Jamie McClelland is with the Centre of Medical Imaging Computing, University College London, London NW1 2BU, UK (email: j.mcclelland@ucl.ac.uk ).

Helen Grimes is with the Department of Radiotherapy Physics, University College London Hospital, London NW1 2BU, UK (email: Helen.Grimes@uclh.nhs.uk ).

Syed Moinuddin is with the Department of Radiotherapy, University College London Hospital, London NW1 2BU, UK (email: syed.moinuddin@nhs.net ).

Ricky Sharma is with the University College London, Cancer Institute, University College London London WC1E 6DD, UK (email: ricky.sharma@ucl.ac.uk ).

Simon Rit is with Univ Lyon, INSA-Lyon, Université Claude Bernard Lyon 1, UJM-Saint Etienne, Centre Léon Bérard, CNRS, Inserm, CREATIS UMR 5220, U1206, F-69373, LYON, France (email: simon.rit@creatis.insalyon.fr).

Kris Thielemans is with the Institute of Nuclear Medicine, University College London, London NW1 2BU, UK (e-mail: k.thielemans@ucl.ac.uk ). images, and this could potentially reduce the accuracy of positional verification.

Currently there are two general approaches for accounting for respiratory motion during $\mathrm{CBCT}$ acquisitions. 1) Respiratory bin gating over the respiratory cycle to form a $4 \mathrm{D}-\mathrm{CBCT}$ data [1], and (2) the use of a respiratory motion model to compensate for motion during the reconstruction [2]. Both methods require a breathing surrogate signal.

The most common method of extracting a breathing signal relies on an external measurement of the respiration [3], for instance, using the Real-Time Position Management (RPM) system (Palo Alto, USA). However, using such a system has some drawbacks such as the need for extra setup, and additional equipment cost, and signals derived from external device may not have a strong and reproducible relationship to internal motion. In contrast, respiratory signals derived from CBCT projection data is more advantageous because it does not require extra setup and eliminates the need for extra equipment cost. Also, signals derived from CBCT projections could potentially have a better correlation to the internal motion.

Our work aims at generating a data driven respiratory motion signal from CBCT projection data. We use techniques based on Principal Component Analysis (PCA) to identify motion that are currently used in other imaging modalities. Other methods for acquiring respiratory signal from CBCT projections exist, such as the Amsterdam shroud [4] and the Local Principal Component Analysis (LPCA) [5], both of these methods greatly rely for the diaphragm to be present within the FOV of the projections in order to obtain a breathing signal.

The potential advantage of our proposed method is that we have the flexibility of applying our approach to ROIs within the projection datasets (e.g. Tumor Region) and this should generate a signal that will be better related to the motion of that particular ROI, instead of a 'global' signal such as that obtained from the AS method or the LPCA technique.

Though PCA so far has been successfully used for motion quantification in PET [6], there are additional challenges in its implementation for $\mathrm{CBCT}$ data, mainly due to the slow rotational motion of the $\mathrm{CBCT}$ scanner, while the PET detectors are stationary. In initial investigations, we will simulate CBCT data of the thoracic region of the body. Finally, we will test our method on a real patient data set. 


\section{METHOD}

\section{Proposed Method}

The proposed method works on the raw projection data. In our method, we can focus on any structure. Here, we chose to focus on the tumor, in contrast to the existing methods mentioned in the section I. To do this, we implemented the tumor enhancement method as described by Martin et al [7]. This method aims at removing the 'non-tumor-region' anatomy from the projections. The resulting data is then passed to PCA. To reduce the influence of the rotational motion, PCA is applied on a subset of the data corresponding to a small range of angles. A sliding window is then used and all data are then combined together at the end. We now give more details about all of the steps.

\section{Tumor enhancement method.}

1) Reconstruct the raw projection data using FDK to obtain an image averaged over respiration. 2) Segment the tumor region from the reconstruction and create the tumor region and non tumor region in image space. 3) Forward project the tumor region and non tumor regions. 4) Subtract the forward projections of the non tumor region away from the initial raw projections. 5) Create a mask of the tumor region, and apply the mask to the output obtained in step 4. Ideally, we should be left with an enhanced projection of the segment region where features in the background which are the stationary have been eliminated.

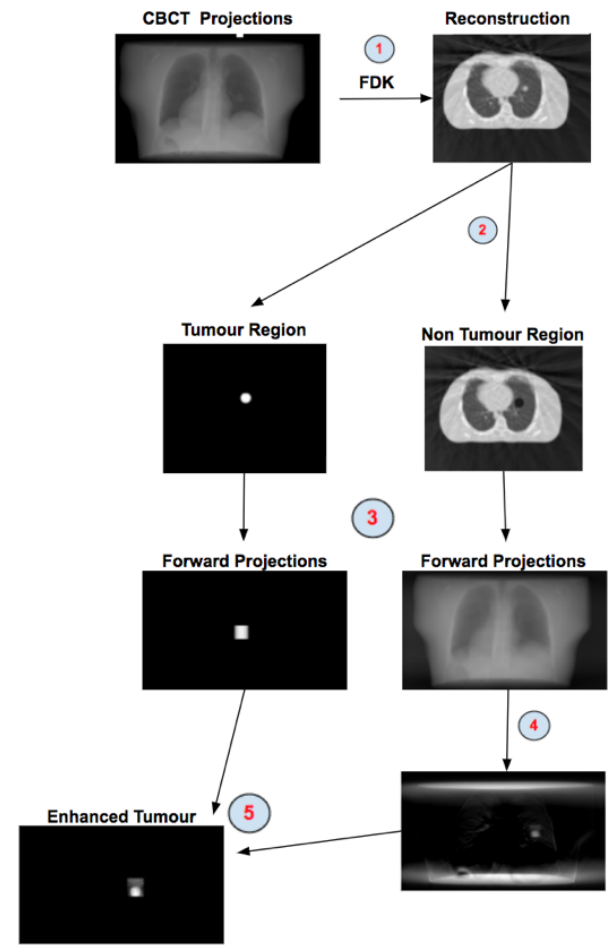

Fig. 1. Layout of the tumor enhancement method.

\section{Application of PCA in sliding windows.}

PCA is a form of dimensionality reduction technique. PCA is a method of identifying patterns in data and expressing the data in a form that highlights their similarities and differences. Here, it is used to describe dynamic data, $\boldsymbol{s}_{\boldsymbol{i}}$ as a linear expansion of $\boldsymbol{K}$ orthogonal principal components. The $\mathbf{1}^{\text {st }}$
Principal Component (PC) weight represents the largest variation in the data.

$$
s_{i} \approx \bar{s}+\sum_{k=1}^{K} w_{i k} P_{k}
$$

$\bar{s}$ is the mean of the data.

$P_{k}$ is the $\mathrm{PC}$

$w_{i k}$ is the weight factor

In order to extract the respiratory trace from the tumor enhanced projection, we applied PCA in a column wise method as shown in figure 2. We computed PCA by choosing 20 time points (corresponding to 20 degrees movement of the detector), we shifted the window size by one angle and computed PCA on each window as illustrated by the different colours in figure 2 . For each window, we computed weights corresponding to the 1st principal component (PC) which represents the largest variation in the data. The PCA signal has an arbitrary sign. Therefore, we compute the Pearson correlation between two adjacent PC weights over the overlapping time points, and, if they are inversely correlated, we change the sign of one weight. As a result of this step, we end up with correlated, weights as shown in figure 2 D. For the final step, we superimpose all the weights together by averaging all weight values available at each time points.

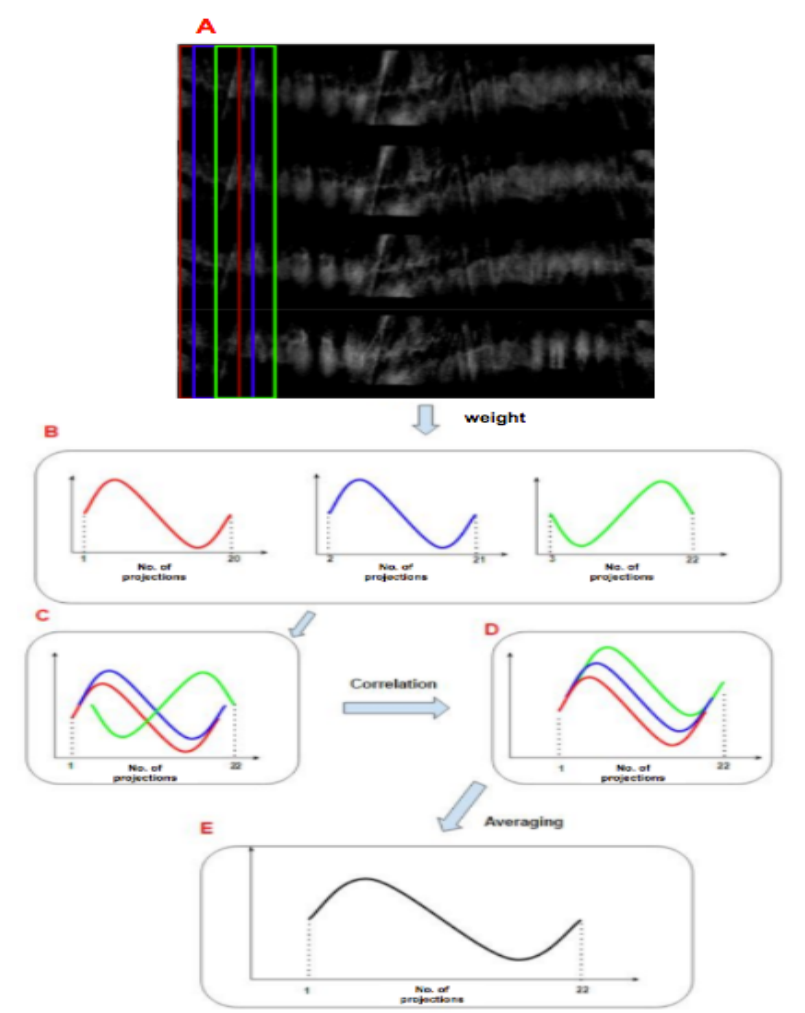

Fig. 2. Layout of our proposed method. A represents the projection data. B shows the weights for the 1st PC for each window (Red, Blue, and Green). C shows the 3 weights in the same space. D shows the weights after applying the correlation. E shows the output after superimposing the 3 weights. 


\section{Simulation}

XCAT is a software package that is commonly used for anatomical modelling for medical imaging research [8]. We used XCAT to simulate CBCT images for the thoracic cavity of the human body where a lesion was inserted into the right region of the thoracic cavity. Using XCAT, we simulated a 4D data set, whereby the first three dimensions represent the $3 \mathrm{D}$ volume of the body and the $4^{\text {th }}$ dimension represents the ground truth respiratory motion which was introduced into the data. The acquisition model parameters for the XCAT simulation were (lesion diameter $=10 \mathrm{~mm}$, slice width $=$ $0.25 \mathrm{~cm}$, pixel width $=0.0976 \mathrm{~cm}$, volume size $=512 \times 512 \times$ 100 ) and the motion was in the Superior Inferior (SI) direction. For the ground truth motion, two motion parameters were used respectively, a periodic sinusoidal signal and a breathing signal acquired from a patient's respiratory trace.

CBCT data were simulated as follows. Projections of the phantom model were obtained with an open source reconstruction software (RTK) [9]. Each 2D projection image represents a specific time point (and therefore detector position) with the corresponding induced motion in the XCAT simulation.

\section{Clinical Data}

We also tested our proposed method on real clinical data. The patient CBCT data was acquired using an on-board imaging (OBI) system (Varian Medical System, Palo Alto, CA). The detector contains $1024 \times 768$ pixels. 683 projections were collected over $360^{\circ}$ (11.3fps). The reconstruction and forward projection steps required for the enhanced tumor method were also performed using RTK [9].

\section{RESULTS}

The respiratory signal obtained from using our proposed method with the simulation data was similar to the ground truth respiratory trace. We extracted a signal based on the simulation setup described in section II (simulation). In addition, included in the results is a signal extracted from a simulation which used a real patient breathing trace as input.

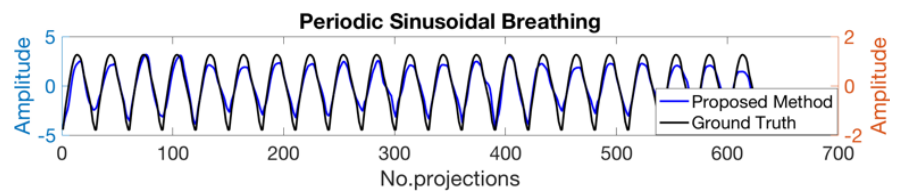

Fig. 3. Respiratory trace obtained using our proposed method and the ground truth respiratory signal introduced into the data. The ground truth signal was a periodic sinusoidal breathing pattern.

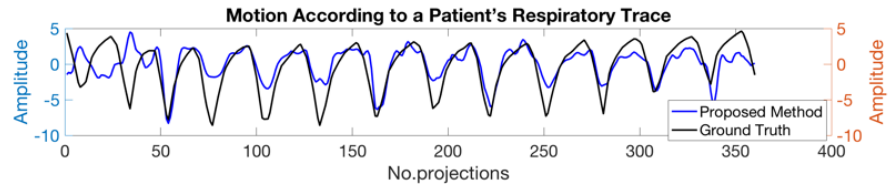

Fig. 4. Comparison between our proposed method and the ground truth input signal. The ground truth signal was a trace of a real patients signal acquired with an external device (RPM). For the first 50 projections, the sign of the signal extracted via our technique seems to be inversely related to the true ground truth signal. The inverse in the sign could potentially be due to a local failure of the the correlation based method described in figure 2 .

The initial result obtained for the clinical data is shown in figure 6 . Further validation is needed.

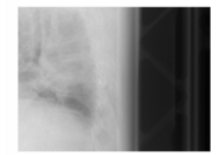

1

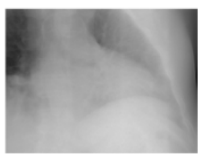

200

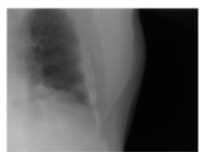

350

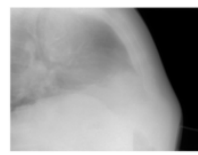

500
Fig. 5. Projections of the clinical dataset.

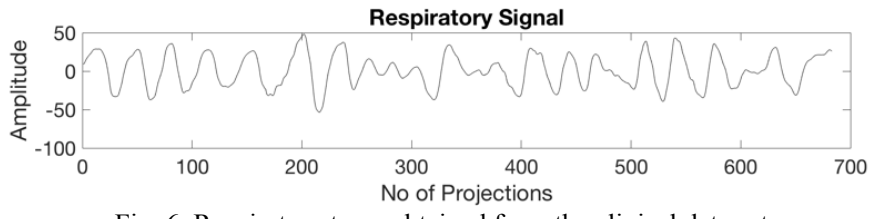

Fig. 6. Respiratory trace obtained from the clinical data set.

\section{CONCLUSION}

We have developed a method of extracting a respiratory signal from CBCT projection data. We evaluated our method on XCAT simulations and real patient data. Our proposed method was able to extract a respiratory signal similar to the ground truth for the periodic input signal and to a lesser extent for the variable motion signal. The difference in amplitude in both respiratory motion cases presented could be due to the presence of detector motion in the input to PCA. Nevertheless, the phase of the respiratory cycle extracted via the proposed method was consistent with the input signal.

In addition, we expect that noise would affect the enhanced tumour region created as well as the PCA signal. This will be investigated in future studies.

Ground truth was not available for the clinical data, thus there was no "true" means of comparison for the respiratory output extracted. However, truncation was an issue for the respiratory signal obtained as seen from the projection images of the clinical data shown in figure 5. The full extent of the data is not covered by the FOV.

We plan on improving our method by applying iterative reconstruction, potentially reducing the effect of truncation on the patient data. We will apply our proposed method to more clinical data. In addition, we will also compare our method to other techniques such as the AS method, and LPCA technique.

\section{REFERENCES}

[1] J. J. Sonke, L. Zijp, P. Remeijer, and M. van Herk, "Respiratory correlated cone beam CT," Med. Phys. 32, 1176-1186, 2005.

[2] S. Rit, J. W. H. Wolthaus, M. van Herk, and J. J. Sonke, "On-the-fly motion-compensated cone-beam CT using an a priori model of the respiratory motion," Med. Phys. 36(6), 2283-2296, 2009.

[3] P. J. Keall P. J. Keall, G. S. Mageras, J. M. Balter, R. S. Emery, K. M. Forster, S. B. Jiang, J. M. Kapatoes, D. A. Low, M. J. Murphy, B. R. Murray, C. R. Ramsey, M. Van Herk, S. S. Vedam, J. W. Wong, Ellen Yorke "The management of respiratory motion in radiation oncology report of AAPM Task Group 76". Med. Phys. 33 3874 - 3900, 2006. 
[4] Zijp, L., Sonke, J.J., Herk, M.V.: "Extraction of the respiratory signal from sequential thorax cone-beam X-ray images". International Conference on the Use of Computers in Radiation Therapy, 2004.

[5] H. Yan X. Wang, W. Yin, T. Pan, M. Ahmad, X. Mou, L. Cerviño, X. Jia, S. B Jiang. "Extracting respiratory signals from thoracic cone beam CT projections". Physics in Medicine and Biology. 581447 - 1464, 2013.

[6] K. Thielemans, S. Rathore, F. Engbrant, P. Razifar. "Device-less gating for PET/CT using PCA". IEEE Nuclear Science Symposium Conference, 3904-3910, 2011.

[7] J. Martin, J. McClelland, C. Yip. "Building motion models of lung tumors from cone-beam CT for radiotherapy applications". Physics in Medicine and Biology, 58:1809-1822, 2013.

[8] W. P. Segars, G. Sturgeon, S. Mendonca, J. Grimes, B. M. W. Tsui. "4D XCAT phantom for multimodality imaging research". Medical Physics Online, 37:4902 - 4915, 2010

[9] M. V. Oliva, S. Brousmiche, R. Labarbe, D. Sarrut, G. C. Sharp. "The Reconstruction Toolkit (RTK), an open-source cone-beam CT reconstruction toolkit based on the Insight Toolkit (ITK)". Journal of Physics: Conference Series, 489, 012079, 2014. 